\title{
Integrative Analysis of Multiomics Data Identified Acetylation as Key Variable of Excessive Energy Metabolism in Hyperthyroidism-induced Osteoporosis Rats
}

Jiaxin Bei

The Second Affiliated Hospital of Guangzhou Medical University

Shaoping Zhu

Guangdong Medical University

Minqun Du

Guangdong Women's and Children's Hospital

\section{Zheng Tang}

Affiliated Hospital of Guangdong Medical University

\section{Cailing Chen}

Affiliated Hospital of Guangdong Medical University

\section{Kevin Yang}

Sun Yat-Sen University

\section{Zhihui Hu}

Affiliated Hospital of Guangdong Medical University

\section{Ying Zhong}

Affiliated Hospital of Guangdong Medical University

\section{Xianhong Zhang}

The Second Affiliated Hospital of Guangzhou Medical University

\section{Wangen Li}

The Second Affiliated Hospital of Guangzhou Medical University

Zhuo qing Hu ( $\sim$ hzq7744@163.com )

The Second Affiliated Hospital of Guangzhou Medical University https://orcid.org/0000-0002-86529196

\section{Research}

Keywords: Hyperthyroidism, Osteoporosis, Acetylation, Metabolism

Posted Date: July 13th, 2021 
DOl: https://doi.org/10.21203/rs.3.rs-699196/v1

License: (c) (1) This work is licensed under a Creative Commons Attribution 4.0 International License. Read Full License

Version of Record: A version of this preprint was published at Journal of Proteomics on December 1st, 2021. See the published version at https://doi.org/10.1016/j.jprot.2021.104451. 


\section{Abstract \\ Background}

The results from the previous experiment have demonstrated that there were occurrence of bone loss and excess metabolism in Hyperthyroidism-induced rats. Thus, there was speculation that there may be an underlying relationship between metabolism and bone loss. In addition, there were past studies showing acetylation influencing metabolism in tissues and diseases. The hypothesis from this case study stated that excessive metabolism was induced upon acetylated vital metabolism enzymes.

\section{Results}

In the case study, a HYP-induced osteoporosis rats model was used and the glucose metabolite was tested through the acetylation of proteins by the mass spectrometer. The results showed that pivotal enzymes of Glycolysis-Tricarboxylic acid cycle-Oxidative phosphorylation were acetyled along with upregulated metabolites. All the acetyly-lysine sites of related enzymes were listed in this article.Our results showed that bone loss in HYP rats accompanied by upregulation of CREB-binding protein (Crebbp, $\mathrm{CBP}$ ). Furthermore, our result indicated that CBP has a close relationship with enhancement of LDHa that promote glucose metabolism.

\section{Conclusions}

Acetylation is the key variable of energy metabolism in hyperthyroid osteoporosis rats, therein, we showed a representation relationship between CBP and LDHa.

\section{Background}

The skeleton is a metabolic organ which undergoes bone remodeling throughout the human lifespan ${ }^{[1]}$. Bone remodeling is a process of bone resorption and bone formation ${ }^{[2]}$. During bone modeling, multinucleated osteoclasts resorb bone and then followed by bone deposition from osteoblasts in order to maintain skeletal mass. The process of bone remodeling requires synthesis of collagen which requires amount of energy or adenosine triphosphate (ATP). Studies showed that glycolysis plays a primary role in generating ATP supplement in bone formation ${ }^{[3-4]}$. The merge of osteoclasts progenitors into a giant multinucleated cell requires energy from oxidative phosphorylation and mitochondrial biogenesis. Previous data had showed that osteoclasts have more mitochondria per surface area than other cells which supports the fact that osteoclasts require huge amount of energy ${ }^{[5]}$.

Studies from the early 1960's showed that bone tissue take huge amount of glucose to achieve physiological activities and produce lactate as a final production regardless of oxygen conditions ${ }^{[6-7]}$. Later studies revealed that progression of induced-osteoblasts in sequential stages of differentiation 
coincided with significant changes in metabolic situation and mitochondrial activity. ${ }^{[8]}$ Furthermore, mature osteoblasts contained a larger number of mitochondria than immature osteoblasts. The number of ATP increased in maturing cultured-osteoblasts during nodule morphogenesis while the rate of glycolysis rose later in mature osteoblasts ${ }^{[8]}$. However, historical studies indicated that bone tissues have a high amount of citrate which take part in the Tricarboxylic acid (TCA cycle) as the key intermediate ${ }^{[9]}$. In osteoporosis, the metabolism in bone cells is extremely close to the bone cells' metabolism in bone remodeling. Currently, the cellular metabolism is the focus for chronic disease investigations. Also, the process of bone remodeling is mediated by hormone, nutrition and aging factors ${ }^{[2]}$. High level of hyperthyroidism is a major factor contributing to osteoporosis, but it is still uncertain whether the level of hyperthyroidism contributes to the imbalance in metabolism from the bone tissues. Thus, the underlying mechanism of the imbalance of metabolism must be explored.

Supported by recent rapid development of epigenetics, protein modification has become a new focus in treating chronic disease. Histone deacetylase (HDAC) inhibitors are now recognized as potential drugs because they can regulate biological responses though gene transcription or epigenetic control. However, little is known about the consequence of epigenetic changes or protein modification in bone cells or tissues. Peter and Ling had demonstrated that there was a decreased amount of HAT1, HDAC6 and GCN5 in the trabecular bone tissue samples obtained from postmenopausal osteoporosis and osteoarthritis patients. The level of acetylation in OPG, RANKL and Wnt pathway was also found to have decreased in these patients as well ${ }^{[10-11]}$. The modifications in osteoporosis-related proteins were also found in different type of Osteocytes ${ }^{[12]}$. As a coactivator, CBP was found in all of the TH-regulated enhancers ${ }^{[13]}$. $\mathrm{CBP}$ is also an acetylase which transferred acetyl group to the lysine residue at the amino terminal of histones, so that there was depolymerization of the chromatin structure. Moreover, CBP stabilized multiple non-histone proteins by acetylation ${ }^{[14]}$.

In our previous results, the energy metabolism via the glycolysis-TCA cycle-oxidative phosphorylation pathway was positively associated with T4-induced bone histomorphometric changes in rats ${ }^{[16]}$. CBP/P300 was an important acetylase that may regulate the activity of enzymes involved in glucose metabolism in the bone tissue. There are 2 aims in our current research: To further study whether acetylation is closely related to the glycolysis-TCA cycle-oxidative phosphorylation pathway energy metabolism through multiomics data and to explore the role of CBP in HYP-induced osteoporosis.

\section{Results}

\subsection{Osteoporosis in Hyperthyroid rats}

There was a tendency that hyperthyroid rats gained weight more slowly compared to rats with CON (Fig. 1B), especially two weeks after treatment. The T3 and T4 were enhanced in HYP rats (Fig. 1C). Also, the hyperthyroid rats had obvious bone loss and increased bone fragility as expected. Parameters of Bone Biochemistry were changed significantly such as break load, maximum load, elastic load and 
stiffness (Fig. 1D). It was shown that Bone Volume/Tissue Volume (BV/TV), Trabecular Thickness (Tb.Th), Trabecular Separation (Tb.Sp) and Structural Model Index (SMI) seemed to have decreased in treated rats. Also, there was significant descent $(p<0.05)$ in Trabecular Number $(T b . N)$, Connection Density (conn-Dens) and Bone Mineral Density (BMD) from the Micro CT scanning results (Fig. 1A,E). All the parameter from CT demonstrated that hyperthyroid rats had bone loss and high bone fragility.

The different expression proteins had been identified in femoral of rats with osteoporosis caused by hyperthyroidism through Liquid Chromatography-Tandem Mass Spectrometry Analysis. A total of 5227 proteins were detected. Among the 5227 proteins, 712 proteins were upregulated and 560 proteins were downregulated in hyperthyroid rats. Enrichment analysis showed that 4871 proteins took part in 284 pathways. Among the 4871 proteins, 1218 proteins were detected to be differently expressed between treated group and CON group. Of the top 20 pathways with the highest number of differential proteins and their proportion, 8 were related to metabolism. Oxidative phosphorylation, Tricarboxylic acid cycle (TCA cycle), Glycolysis pathways were ranked at 6th and 15th, and 21 st respectively. According to GeneOntology enrichment analysis, there was a total of 195 protein related to production process of precursor metabolites and energy ${ }^{[16]}$. In this research, a HYP-induced OP model was built and then the femur tissue was separated for Acetylated proteomics Analysis of Lysine-Acetylated Proteins. The metabonomics were targeted on TCA, Glycolysis and Oxidative phosphorylation as well(Fig. 2).

\subsection{Acetylome}

A total of 892 nonredundant proteins, 3189 peptides and 914 lysine acetylation sites were identified in the femur of hyperthyroid rats. Among those lysine acetylation sites, 690 sites were upregulated and 66 sites were downregulated (Fig. 3A). 451 lysine-acetylated proteins were identified only in femurs from hyperthyroid rats compared with 45 lysine-acetylated proteins in control rats. At the same time, 238 proteins were found in high level of acetylation and 21 proteins were found in low level of acetylation from the hyperthyroid rats (Supplementary table 1). It was also observed that a significant acetylation occurred in Histone $2(\mathrm{H} 2)$, Histone $3(\mathrm{H} 3)$, Nicotinamide nucleotide transhydrogenase (Nnt) and Nicotinamide adenine dinucleotide (NADH) in HYP rats (Fig. 3B). Also, the acetylation level were promoted in actin, Myosin light chain (MLC1/MLC2), H2B, CREB-binding protein (CREBBP), Glutamate dehydrogenase 1 (Glud1) and nicotinamide adenine dinucleotide phosphate (NADP) in hyperthyroid rats, however no significant acetylation were seen in control rats (Fig. 3C). Furthermore, KEGG pathway enrichment analysis showed that lysine-acetylated proteins (rich factor $(\mathrm{RF})=0.95$ ) were related to glycolysis gluconeogenesis pathway (Fig. 4A).

Gene ontology (GO) analysis mainly include the analysis of biological process (BP), Cellular components (CC) and Molecular Function (MF). The results revealed that lysine-acetylated proteins are highly relevant to Polymenic cytoskeletal fiber $(\mathrm{RF}=0.85)$ in aspect of $\mathrm{CC}$. They are also highly relevant to oxidoreduction coenzyme metabolic process $(\mathrm{RF}=0.92)$ in the aspect of $\mathrm{BP}$. The lysine-acetylated proteins were highly relevant to NAD binding as well $(R F=0.93)$ in the aspect of MF (Fig. 4B). 
The comparison of identified lysine-acetylated proteins and different abundance proteins was summarized in distinct biological metabolic pathway such as TCA, Glycolysis and Oxidative phosphorylation. It is found that some enzymes have been synchronously acetylated and they were expression-regulated in biological metabolic pathway as shown in Fig. 4C.

\subsection{Targeted Metabolomics}

The detection method covered 31 important metabolites in the TCA, glycolysis pathway and oxidative phosphorylation process. TCA and glycolysis metabolites were targeted by the targeted metabolite detection. The cluster diagram showed that the metabolites between two groups can be separated, indicating that the selected candidate metabolites are accurate (Fig. 5A, Supplementary table 2). Final results showed that metabolites in the process of glycolysis, dihydroxyacetone phosphate, $\beta$-D-fructose-6phosphate, lactate and D-glucose-6-phosphate were promoted while metabolites in TCA cycle including oxaloacetate, aconitate, fumarate and L-malic acid were increased compared with CON group (Fig. 5B, Supplementary table 2).

\subsection{Acetylation in glycolysis}

The fructose-bisphosphate aldolase A (Ec 4.1.2.13), Phosphoglycerate kinase 1 (PGK1), Phosphoglycerate mutase 1/2 (PGM1/2), $\alpha / \beta$ enolase ( $\alpha / \beta$ ENO), Pyruvate kinase (PKM) and L-lactate dehydrogenase $A$ chain $(\mathrm{LDH})$ had obvious changes in protein abundance level during glycolysis. They were also acetylated and upregulated in level of protein expression, except for Triosephosphate isomerase (TPI). Combine with the results of Metabolomics, it can be seen that metabolites of glycolysis were significantly increased while major enzymes were synchronously acetylated. The exact acetylation sites of lysine of various enzymes are marked in Fig. 5. Generally, it is positive for the acetylation of enzymes of glycolysis to promote the process in HYP-induced OP rat (Fig. 6).

\subsection{Acetylation in TCA}

In the TCA cycle, proteins that are up-regulated included Citrate synthase, ATP-citrate synthase, Malate dehydrogenase, Succinate-CoA ligase subunit, and NADP. However, Citrate synthase, Malate dehydrogenase, Succinate -CoA ligase subunit $\beta$, and NADP were different in acetylation level. In HYP rats, the level of acetylation was increased in Citrate synthase; the acetylation sites of Citrate synthase were K103, K215, K327 and K105. The level of acetylation in Malate dehydrogenase increased; the acetylation sites of Malate dehydrogenase were K301/280 and K307/286, acetyl. The level of acetylation of Succinate-CoA ligase subunit $\beta$ was increased and decreased; the acetylation site of Succinate-CoA ligase subunit were K75/K73/K70/K28. Also, the level of acetylation in NADP increased; NADP had different levels of different sites. The level of acetylation of K155 and K384 also was rising, but the level of acetylation for $\mathrm{K} 45$ was falling. Among the circulating metabolites, the most obvious difference was seen in Aconitate, Oxaloacetate, Fumarate, L-Malic acid, and Succinate (Fig. 6).

\subsection{Acetylation in oxidative phosphorylation}


According to the results of acetomics, only a few numbers of component had occurrence of acetylation while other components had not. The main reasoning was that one component acetylated could be a competent for functional regulatory. As shown in Fig. 6 and Supplementary table 1, A-H component(Fig. 6) of complex I, III, IV, V were acetylated to accelerate oxidative phosphorylation intramembrane of mitochondria.

\subsection{Close relationship between CBP and LDHa in the promotion of glucose metabolism in Hyperthyroidism-induced Osteoporosis}

The iTRAQ results were confirmed with the western blot analysis which show that citrate syntheses (CS), oxoglutarate dehydrogenase complex (ogdh) and Oxalosuccinate decarboxylase A (Idh3a) were key enzymes in TCA. In addition, mitochondrial ATP synthase subunit 5A (Atp5a1) and Cytochrome c oxidase subunit $5 \mathrm{a}(\mathrm{Cox} 5 \mathrm{a})$ were key proteins for oxidative phosphorylation. Fructose 6 phosphate kinase (PFKM) and Lactate dehydrogenase (LDHa) were also catalytic enzymes in glycolysis. Enzymes in glycolysis-TCA cycle-oxidative phosphorylation pathway in HYP rats were significantly more up-regulated compared to those in $\mathrm{CON}$ rats. Furthermore, the western blot results were consistent with high-throughput sequencing ${ }^{[16]}$.

According to the previous results, CREB-binding protein (CBP) was significantly expressed in HYP rats ${ }^{[14]}$. CBP acted as crucial regulator of energy homeostasis and also played a potential role in mammalian metabolic homeostasis in response to nutrients ${ }^{[17-18]}$. Thus, CBP was investigated in how it influenced glycolysis-TCA cycle-oxidative phosphorylation pathway in HYP rats. HYP rats expressed more CBP compared to $\mathrm{CON}$ rats, and proteins which have interaction with $\mathrm{CBP}$ were detected as one strong band between the range of $55 \mathrm{kD}$ and $70 \mathrm{kD}$ (Fig. 7A).

In mass spectrometry analysis from Shanghai Applied Protein Technology, proteins had to have the Score Sequest $H T \geq 1.5$ as well as having the contaminated proteins and high molecular weight proteins to be eliminated. A total of 53 proteins was identified in the bone tissue of HYP rats and 29 proteins were identified in the CON ones. The intersection showed that 24 proteins were found in both groups while 29 proteins were only found in the HYP rats and 5 proteins were only found in the CON rats. After unclear proteins were removed, there was protein profile identification of the two groups of IP (Fig. 7A and Tab S3 ). 6 catalytic enzymes related to glycolysis-TCA-oxidative phosphorylation were identified in HYP rats and 5 catalytic enzymes related to glycolysis-TCA-oxidative phosphorylation were identified in CON rats. Among these proteins, there are 4 enzymes been identified in both groups including L-lactate dehydrogenase A chain LDHa (ID: P04642), Pyruvate kinase PKM (ID: P11980), ATP synthase subunit alpha (ID: F1LP05), and ATP synthase subunit beta (ID: G3V6D3). From the previous multi-omics analysis, LDHa (ID: P04642) level was increased and was also upregulated in multiple sites of acetylation. The colP showed that LDHa had an interaction with CBP and that there was a significant upexpression of LDHa in HYP rats (Tab S3). 
The results showed that $T 3\left(10^{-7} \mathrm{M}\right.$ and $\left.10^{-8} \mathrm{M}\right)$ promoted the expression of CBP and LDHa in MG63 cells (Fig. 7B). However, there was no increase in LDHa level in CBP-knockdown MG63 cells (shCBP-MG63 cells) after T3 treatment (Fig. 7C). A-485 is an inhibitor of CBP, so it can inhibit the acetylation activity of both $\mathrm{CBP}$ and $\mathrm{p} 300^{[40]}$. When the rats were pre-treated with $\mathrm{A}-485$, there was an up-expression of LDHa induced by T3(Fig. 7D).

There was no differences in MG63 cell viability between all concentrations of T3 $\left(10^{-5}-10^{-8} \mathrm{M}\right)$ (Fig S1A ). After induction with different concentrations of T3 for 3 or 6 days, there was a significant increase in ALP activity assay (Fig S2A). OCN assay results showed that T3 began to stimulate the accumulation of $\mathrm{OCN}$ in the supernatant on the 6th day(Fig S2B). However, the lactate release had been induced by $\mathrm{T} 3$ since the second day(Fig S2C). These mentioned above scenario were significantly reversed in shCBPMG63 cells (Fig S1B, Fig S2D-F). MG63 cells were treated with $10^{-7} \mathrm{~mol} / \mathrm{L}$ and $10^{-8} \mathrm{~mol} / \mathrm{L} \mathrm{T3}$ for 3-9 days and after pre-treatment of $10^{4} \mathrm{nM}, 10^{2} \mathrm{nM}$ and $1 \mathrm{nM}$ of A-485, the ALP assay results showed that $10^{4} \mathrm{nM}$ and $10^{2} \mathrm{nM}$ of A-485 had inhibited the cell ALP activity (Fig S1C-D ).

A: NADH dehydrogenase 1 alpha subcomplex subunit 9

B: NADH-ubiquinone oxidoreductase75 kDa subunit

C: Cytochrome b-c1 complex subunit 6

D: Cytochrome c oxidase subunit 4 isoform 1

E: ATP synthase $F(0)$ complex subunit B1

F: ATP synthase subunit alpha

G: ATP synthase subunit d

$H$ : ATP synthase subunit $f$

Fig S1. (A) Effect of T3 on cell viability; (B) Knockdown was quantified by quantitative PCR; (C) Effect of A-485 on cell viability; (D) Effect of A-485 on ALP activity.

Fig S2 The effect of T3 and CBP on osteogenic phenotype in MG63 cell. (A) Cellular ALP activity; (B) ELISA analysis of OCN secretion in MG63; (C) Extracellular lactate in MG63 cell; (D) Cellular ALP activity in shCBP cell ; (E) Extracellular lactate in shCBP cell; (F) ELISA analysis of OCN secretion in shCBP cell.

\section{Discussion}

It was confirmed that the weight was significantly lower in hyperthyroid rats compared to the control groups ${ }^{[14]}$. The HYP rats gain weight more slowly than the control group as well. In addition, the weight loss was closely related to energy consumption. At the same time, bone loss was a significant 
phenomenon in HYP rats which can be seen in the decrease of BV/TV, Tb.Th, Tb.Sp, Tb.N, conn-Dens, BMD and SMI from the Micro CT scanning results. The results of bone loss from this study was the same as the previous study [14]. The former results showed that all the key enzymes in the glycolysis-TCA cycleoxidative phosphorylation pathway were upregulated as well as all the enzymes that contributed to the process of ATP production. Bone remodeling consumed numerous ATP from glucose catabolism $[3,5]$, especially in high bone turnover osteoporosis. In addition, the thyroid hormone (TH) played a vital role in energy metabolism since high load of TH enhance the metabolism of body substances. It was found that T4 had a positive effect on bone formation, differentiation, and mineralization in osteoblasts ${ }^{[19,20]}$. Also, excessive T4 levels might promote both osteoclast and osteoblast bioenergetics by strengthening the oxidative phosphorylation-TCA cycle- glycolysis pathway ${ }^{[14]}$. However, the mechanism of excessive glucose metabolism of bone tissue in HYP-induced OP rats is still unknown.

The study showed that more than half of proteins detected were found to be acetylated, containing 914 actyl sites and most of them were upregulated. Early research had indicated that each different organ in rats had different level of acetylation ${ }^{[21]}$. However, this study explores the relationship of acetylation and bone tissue of HYP-induced OP rats which the former studies did not explore. Among these acetylproteins, the most conspicuous ones are metabolism-related proteins, skeletal muscle-related protein and histone which hinted the fact that acetylation might be a bridge between excessive energy metabolism and osteoporosis. KEGG pathway analysis indicated that majority of acetyl-proteins were involved in glycolysis gluconeogenesis pathway which became corroborative evidence supporting the claim that acetylation is a vital factor in HYP-induced OP. Also, acetylation had emerged as a key regulatory mechanism in cellular metabolism. The three mitochondrial KATs which were GCN5L1, KAT8, ACAT1 have been reported as important factor in mediating the mitochondrial protein acetylation ${ }^{22-25]}$. The results from study accorded with the opinion above that from GO analysis, the lysine-acetylated protein took part in oxido-reduction coenzyme metabolic process which mainly happened in mitochondria. This finding confirmed that acetyl-proteins had a critical effect on metabolism and there were acetyl-proteins in bone tissue in HYP-osteoporosis. The overlap of acetyl-proteins and metabolism-related proteins were analyzed and among the expression-different proteins, there were several unique proteins from Glycolysis- TCA- Oxidative phosphorylation. Thus, a claim was made that acetylation in metabolismrelated proteins is the key point to promote the level of energy metabolism in HYP-osteoporosis rats.

Metabolomics is an interdisciplinary discipline that emerged in the post-genomics era, reflecting the multiple dynamic responses of metabolite levels. Metabolomics was used of it to explore the relationship of Glycolysis- TCA- Oxidative phosphorylation in HYP-osteoporosis rat. All the results demonstrated that some final products from Glycolysis- TCA- Oxidative phosphorylation were increased and the results were in line with the results from the previous study. However, from these results, it cannot be confirmed whether elevating the level of AC in bone could enhance the energy metabolism of bone because different changes of the level of AC happened in separate sites of proteins. To some extent, most of them were upregulated except K307/286 in MDH and K45 in NADP. 
Shimin Zhao et al. identified four acetylated lysine (K185, K301,K307, K314) in acetylation of MDH. They were enhanced by $60 \%$ when Chang liver cells were exposed to high dose of glucose ${ }^{[26]}$. From the study, the same outcome was found in the acetylation sites of Malate dehydrogenase: K301/280 and K307/286. Therefore, Malate dehydrogenase-catalyzed chain reaction in HYP-induced OP rats is to some extent similar to that of high-dose-glucose-induced. ICDH2 (isocitrate dehydrogenase 2, a key regulator of this metabolic cycle) fraction of the partially purified protein was also acetylated in a feeding-dependent manner and deacetylated by SIRT3 ${ }^{[27]}$. However, the activation was decreased after acetylation in wildtype strain YPH499 and they could be seen in liver, inner ear and brain as well ${ }^{[27-28]}$. On the other hand, Sirt3 directly deacetylated and activated mitochondrial Idh2 in response to caloric restriction which caused increased NADPH levels in mice ${ }^{[29]}$. Taken together, it was confirmed that HYP-induced OP is a kind of metabolic disease consuming a mass of energy, in contrast to caloric restriction. Almost all the final production of TCA were increased in HYP-induced OP rats which showed that the cycle was more active than controls.

Nicotinamide Adenine Dinucleotide $\left(\mathrm{NAD}^{+}\right)$is a fundamental molecule in redox signaling and meanwhile NADH is in the reduced form NAD ${ }^{+}$. NADPH (Nicotinamide Adenine Dinucleotide Phosphate) is a coenzyme that acts as a reducing agent in most reactions, but mainly in anabolic reactions. The redox state of $\mathrm{NAD}^{+} / \mathrm{NADH}$ or $\mathrm{NADP}^{+} / \mathrm{NADPH}$ is the best parameter to characterize mitochondrial function in vivo [30]. However, the level of $\mathrm{NADP}^{+} / \mathrm{NADPH}$ or $\mathrm{NADP}^{+} / \mathrm{NADPH}$ was not tested but it could be seen that there was a decline of $\mathrm{NADP}^{+}$in HYP-induced OP rats. $\mathrm{NADP}^{+}$works as an untypical co-enzyme which catalyzes the conversion among Isocitrate, Oxalosuccinate and 2-Oxaglutarate in TCA (Fig. 5). The condition of oxidative stress in HYP-induced OP rats could not be speculated due to the absence of the change of $\mathrm{NADPH}$. There was also a decline of $\mathrm{NADP}^{+}$which had a passive effect on collateral reaction in TCA. The $\mathrm{NADP}^{+}$were also acetylated as well in disparate lysine sites with opposite change in acetylation level, so there is mystery behind the correlation between acetyl-NADP ${ }^{+}$and activated TCA. Many molecules involved in main reaction of TCA were found to be acetylated to promote TCA, but that of collateral coenzyme were known little. Moreover, pentose phosphate pathway (PPP) is the source of NAPDH ${ }^{[31]}$. The acetylation and reductive production of NAPD might have some correlation with PPP though we still need evidence to proof it.

Furthermore, glycolysis plays a vital role in the differentiation process of osteoclasts and glycolysis is the main energy source for immature osteoblasts ${ }^{[8]}$. Combine with results from the previous study, overload of HYP had induced high turnover type bone loss ${ }^{[16]}$. Also, many precursor osteoclasts had differentiate into mature osteoclasts which means they consume more energy from glycolysis. A summary analysis also showed that estrogen deficiency promote glycolysis and glycolytic-coupled TCA cycle function ${ }^{[32] .}$

Former researches had pointed out that acetylation of pyruvate kinase (PK) played a regulatory role in glycolysis. The acetylation at K305 significantly decreased its' activity which reduced glycolysis in muscle ${ }^{[33]}$. Ariely Barbosa Leite et $a^{[34]}$ also found that activity of fructose-bisphosphate aldolase (Aldo) 
was also reduced with acetylation at K157 in Trypanosoma brucei, but deacetylation in vitro partial recovered it. Moreover, the acetylation of K147 of Aldo B, which is mainly located in liver of rats, appeared to abolish the enzymatic activity of Aldo B because K147 as well as the surrounding amino acids was highly conserved as a substrate binding site in various species ${ }^{[21,35]}$. In contrast, acetylation of most enzymes in glycolysis promoted glycolysis in HYP rats like what we show in Fig. 5. Zhao D et al ${ }^{[36]}$ demonstrated that total 8 lysine sites would be acetylated in LDH-A which catalyzed lactate in glycolysis. The LDH-A was acetylated at lysine 5 (K5) and the acetylation inhibited LDH-A activity by reducing the protein level in pancreatic cancers. However, according to the mass-spectrometry analysis from this stud, 2 putative acetylation sites were identified which were K126 and K64. In addition, the protein level and the production of LDH-A in HYP rats were enhanced which indicated that acetylation at different sites may cause distinct biofunction. Unlike tumor intracellular environment, acetylation of enzymes of glycolysis in HYP rats contributed to the promotion of energy metabolism, but there was no proof that it changed the approach to energy supplement. As for the change of triosephosphate isomerase (TPI), it was speculated that it is not typical for the excessive energy metabolism to be present in HYP-OP rats because it cannot contribute to the total production of downstream metabolite-glycerone-3-phosphate. It also catalyzed the dihydroxyacetone phosphate to become glycerone-3-phosphate, but upstream-metabolite $\beta$-D-fructose 6 phosphate can transform into glycerone-3-phosphate directly.

In an early study, it was reported that acetylation in TPI, ENO and PGK affected the activities of these enzymes in live mouse ${ }^{[37]}$. Combined with results from this study, TPI, ENO and PGK were acetylated to enhance the glycolysis in HYP-induced OP rats. Even in some microorganism, such as Trypanosoma brucei, acetyl-ENO regulated the process of glycolysis ${ }^{[34]}$. The complexes in oxidative phosphorylation in many plants could be acetylaed, S-nitrosylated and phosphorylated ${ }^{[34]}$. Another research indicated that immature osteoblasts utilized oxidative phosphorylation and aerobic glycolysis during the differentiation [39]. According to previous results, HYP-induced OP is a kind of high bone turnover osteoporosis ${ }^{[16]}$ which is consistent with the study that oxidative phosphorylation was increased to generate enough ATP for bone remodeling. Also, many diseases might occur along with abnormal oxidative phosphorylation such as excessive mitochondrial metabolic caused by acetylation of enzymes in alcoholic kidney diseases [40] and acetylation of mitochondrial transcription factor-induced reductive mitochondrial oxidative phosphorylation in diabetes in heart disease rats ${ }^{[41]}$. As mentioned above, the deacetylation of some sites of certain proteins might have completely opposite function in different condition. In this study, it was speculated that acetylation of related-enzymes mainly increased activity of oxidative phosphorylation in HYP-induced OP rats. Unlike tumor tissue, hyperactive oxidative phosphorylation, instead of aerobic glycolysis, might be the primary energy supplement for both bone resorption and bone formation in bone remodeling. However, it is hard to figure out which component was dominant in affecting oxidative phosphorylation since different components of complexes might occur at different posttranslational modification including acetylation during excessive activities ${ }^{[2,42]}$.

This experiment used CO-IP and mass spectrometry to identify proteins which has interaction with CBP. Experiments in vitro cell show that $10^{-7} \mathrm{~mol} / \mathrm{L}$ and $10^{-8} \mathrm{~mol} / \mathrm{L} \mathrm{T3}$ promoted the cellular ALP activity and 
osteocalcin secretion, also stimulated expression of LDHa and secretion of lactate. But it showed a inhibition after knocking down CBP in T3 administration. CBP/P300 is an important acetylase and transcription factor for thyroid hormone signaling. Some studies have reported that $\mathrm{CBP} / \mathrm{p} 300$ regulates the homeostasis of energy metabolism through its acetyltransferase activity and transcriptional coactivator $^{[14]}$. There is one speculation that CBP itself is a downstream target that is activated in response to mitochondrial stress. Such as changes in mitochondrial metabolism may modulate the accumulation of acetyl-CoA, which acts as a substrate for the acetyltransferase activity of $\mathrm{CBP} / \mathrm{p} 300^{[43]}$. Meanwhile, CBP acts on SMAD3, $\beta$-catenin, HIF- $a$, and NF-KB to stimulate fbrogenic genes expression ${ }^{[44,45]}$, whereas HIF-1a and $\beta$-catenin have a close relationship with bone repair ${ }^{[46]}$. These hints $C B P$ may play a role in bone metabolism in some certain way. Our data demonstrated that CBP has interaction with LDHa to promote glycolysis, although the exact underlying mechanism between them is not demonstrated. As mentioned in the literature review, CBP impacted on different genes or proteins in two different ways, respectively, which mediate targets acetylation or promote transcription of targets ${ }^{[45]}$. Similarly, LDHA may be affected by CBP in different ways mentioned above. Very little was found in the literature on the question of correlation between CBP and LDHa in HYP rats. Undeniable, it is a limitation that the manner in which CBP positively interacts with LDHa has yet to be elucidated in our research.

\section{Conclusion}

The posttranslational modification such as acetylation, phosphorylation, and ubiquitination had played a vital role in mediating energy metabolism. According to this study, acetylation is key to excessive energy metabolism in HYP-induced osteoporotic rats. CBP as a acetylase played an important role in energy metabolism especially in the interaction with LDHa to promote glycolysis. However, the underlying mechanism between acetylation of pivotal enzymes and changes of energy metabolism need to be explored to fully understand the relationship between acetylation and energy metabolism in HYP-induced osteoporotic rats.

\section{Declarations}

\section{Ethics approval and consent to participate}

This study was approved by the clinical research institution review committee and ethics review committee of the Affiliated Hospital of Guangdong Medical University.

\section{Availability of data and materials}

The datasets used and/or analysed during the current study are available from the corresponding author on reasonable request.

\section{Consent for publication}


We confirm that this manuscript is original. If accepted, the article will not be published elsewhere in the same form, in any language, without the written consent of the publisher.

\section{Conflicts of interests}

The authors declare that they have no competing interests.

\section{FUNDING}

This research was supported by the grants from China Postdoctoral Science Foundation Grant (Grant NO. 2019 M652859).

\section{Author Contribution}

Zhuoqing Hu and Wangen Li made substantial contributions to the conception and design, analyzed and interpreted the data, performed the literature search, and was the major contributor in the writing of the manuscript. Zheng Tang, Zhihui Hu, Xu Chen and Ying Zhong made substantial contributions to the conception and design and revised the draft critically for important intellectual content. Jiaxin Bei, Shaoping Zhu, Cailing Chen, Minqun Du and Kevin Yang made substantial contributions to the conception and design, analyzed and interpreted the data, and revised the draft critically for important intellectual content. All authors read and approved the final manuscript.

\section{Acknowledgements}

None

\section{References}

[1] Raisz, L.G. Physiology and pathophysiology of bone remodeling. Clin. Chem. 1999, 45, 1353-1358.

[2] Wen-Chih Lee, Anyonya R. Guntur, Fanxin Long et al. Energy Metabolism of the Osteoblast: Implications for Osteoporosis. Endocrine Reviews, 2017, 38(3):255-266.

[3] Indo Y, Takeshita S, Ishii KA et al. Metabolic regulation of osteoclast differentiation and function. J Bone Miner Res. 2013; 28(11):2392-2399.

[4] Ahn H, Lee K, Kim JM et al. Accelerated Lactate Dehydrogenase Activity Potenti- ates Osteoclastogenesis via NFATc1 Signaling. PLoS One. 2016;11(4):e0153886.

[5] Lemma S, Sboarina M, Porporato PE et al. Energy metabolism in osteoclast formation and activity. Int J Biochem Cell Biol. 2016;79:168-180.

[6] Borle AB, Nichols N, Nichols G Jr. Metabolic studies of bone in vitro. The Journal of biological chemistry.1960, 235:1206-1210. 
[7] Cohn DV, Forscher BK. Aerobic metabolism of glucose by bone. The Journal of biological chemistry. 1962, 237:615-618.

[8] Komarova SV, Ataullakhanov Fl, Globus RK. Bioenergetics and mitochondrial transmembrane potential during differentiation of cultured osteoblasts. Am J Physiol Cell Physiol. 2000; 279(4):C1220-C1229.

[9] Costello LC, Franklin RB, Reynolds MA et al. The Important Role of Osteoblasts and Citrate Production in Bone Formation: "Osteoblast Citration" as a New Concept for an Old Relationship. Open Bone J. 2012;4: 27-34.

[10] Vrtačnik, P., Zupan, J., Mlakar, V et al. Epigenetic enzymes influenced by oxidative stress and hypoxia mimetic in osteoblasts are differentially expressed in patients with osteoporosis and osteoarthritis. Sci Rep 8, 16215 (2018).

[11] Jing Huan, Xiaoxia Su, Bo Gao et al. Epigenetic inhibition of Wnt pathway suppresses osteogenic differentiation of BMSCs during osteoporosis. Cell Death and Disease (2018)9:176.

[12] Bora Faulkner, Kristina Astleford, Kim C. Mansky. Regulation of Osteoclast Differentiation and Skeletal Maintenance by Histone Deacetylases. Molecules 2019, 24, 1355; doi:10.3390.

[13] Shabtai Y, Nagaraj NK, Batmanov K, Cho YW, Guan Y, Jiang C, Remsberg J, Forrest D, Lazar MA. A coregulator shift, rather than the canonical switch, underlies thyroid hormone action in the liver. Genes Dev. 2021 Mar 1;35(5-6):367-378. doi: 10.1101/gad.345686.120. Epub 2021 Feb 18. PMID: 33602873; PMCID: PMC7919419.

[14] Lin CC, Kitagawa $M$, Tang $X$, et al. CoA synthase regulates mitotic fidelity via CBP-mediated acetylation. Nat Commun. 2018;9(1):1039. Published 2018 Mar 12. doi:10.1038/s41467-018-03422-6.

[15] Sien Lin, Wayne Y.W. Lee, Meiling Huang et al. Aspirin prevents bone loss with little mechanical improvement in high-fat-fed ovariectomized rats. European Journal of Pharmacology 791 (2016) 331338.

[16] Hu Z, Du M, Lai W et al. Energy Metabolism in the Bone is Associated with Histomorphometric Changes in Rats with Hyperthyroidism. Cell Physiol Biochem 2018;46:1471-1482.

[17] Yao W, Wang T, Huang F. p300/CBP as a Key Nutritional Sensor for Hepatic Energy Homeostasis and Liver Fibrosis. Biomed Res Int. 2018 May 15;2018:8168791. doi: 10.1155/2018/8168791. PMID: 29862292; PMCID: PMC5976926.

[18] Zhou F, Liu Q, Zhang L, et al. Selective inhibition of CBP/p300 HAT by A-485 results in suppression of lipogenesis and hepatic gluconeogenesis. Cell Death Dis. 2020;11(9):745. Published 2020 Sep 11. doi:10.1038/s41419-020-02960-6 
[19] Scarlett A, Parsons MP, Hanson PL et al. Thyroid hormone stimulation of extracellular signalregulated kinase and cell proliferation in human osteoblast-like cells is initiated at integrin alphaVbeta3. J Endocrinol. 2008;196:509-517.

[20] Cheng S, Xing W, Pourteymoor S et al. Effects of thyroxine (T4), 3, 5,3'-triiodo-L-thyronine (T3) and their metabolites on osteoblast differentiation. Calcif Tissue Int. 2016;99:435-442.

[21] Alicia Lundby, Kasper Lage, Brian T. Weinert et al. Proteomic Analysis of Lysine Acetylation Sites in Rat Tissues Reveals Organ Specificity and Subcellular Patterns. Cell Reports, 2012,2,419-431.

[22] Chatterjee A, Seyfferth J, Lucci J et al. Acetyl Transferase Regulates Transcription and Respiration in Mitochondria. Cell 2016, 167, 722-738 (e23).

[23] Scott I, Webster BR, Li JH et al. Identification of a Molecular Component of the Mitochondrial Acetyltransferase Programme: A Novel Role for Gcn5l1. Biochem. J 2012, 443, 655-61.

[24] Scott I, Webster BR, Chan CK et al. Gcn5-Like Protein 1 (Gcn5l1) Controls Mitochondrial Content through Coordinated Regulation of Mitochondrial Biogenesis and Mitophagy. J. Biol. Chem 2014, 289, 2864-72.

[25] Fan J, Shan C, Kang HB et al. Tyr Phosphorylation of Pdp1 Toggles Recruitment between Acat1 and Sirt3 to Regulate the Pyruvate Dehydrogenase Complex. Mol. Cell 2014, 53, 534-48.

[26] Shimin Zhao, Wei Xu, Wenqing Jiang et al. Regulation of cellular metabolism by protein lysine acetylation. Science, 2010,327, 1000-1004.

[27] Christine Schlicker, Melanie Gertz, Panagiotis Papatheodorou et al. Substrates and Regulation Mechanisms for the Human Mitochondrial Sirtuins Sirt3 and Sirt5, Journal of Molecular Biology, 2008, 382(3),790-801.

[28] Someya, S. Wei Yu,3,5 William C. Hallows et al. Sirt3 mediates reduction of oxidative damage and prevention of age-related hearing loss under caloric restriction. Cell. 2010,143, 802-812.

[29] Shinichi Someya, Wei Yu, William C. Hallows et al. Sirt3 Mediates Reduction of Oxidative Damage and Prevention of Age-related Hearing Loss under Caloric Restriction

Cell. 2010, 24; 143(5): 802-812.

[30] Wu, Jinzi, Jin, Zhen, Zheng, Hong et al. Sources and implications of NADH/NAD+

Redox imbalance in diabetes and its complications. Diabetes, Metabolic Syndrome and Obesity: Targets and Therapy 2016:9, 145-153.

[31] Esen, Emel囚Long, Fanxin. Aerobic Glycolysis in Osteoblasts. Curr Osteoporos Rep. 2014; 12(4): 433438. 
[32] Donatella Granchi, Nicola Baldini, Fabio Massimo Ulivieri et al. Role of Citrate in Pathophysiology and Medical Management of Bone Diseases. Nutrients 2019, 11, 2576.

[33] Y. Xiong, Q-Y. Lei, S. Zhao et al. Regulation of Glycolysis and Gluconeogenesis by Acetylation of PKM and PEPCK. cold Spring Harb Symp Quant Biol. 2011, 76: 285-289.

[34] Ariely Barbosa Leite, Antoniel Augusto Severo Gomes, Ana Caroline de Castro Nascimento Sousa, Marcos Roberto de Mattos Fontes, Sergio Schenkman, Nilmar Silvio Moretti; Effect of lysine acetylation on the regulation of Trypanosoma brucei glycosomal aldolase activity. Biochem J .2020, 477 (9): 17331744.

[35] St-Jean, M., Blonski, C., and Sygusch, J et al. Charge stabilization and entropy reduction of central lysine residues in fructose-bisphosphate aldolase. Biochemistry. 2009, 48, 4528-4537.

[36] Zhao D, Zou SW, Liu Y, et al. Lysine-5 acetylation negatively regulates lactate dehydrogenase $A$ and is decreased in pancreatic cancer. Cancer Cell 2013;23:464-76.

[37] Kim SC, Sprung R, Chen Y, et al. Substrate and functional diversity of lysine acetylation revealed by a proteomics survey. Mol Cell. 2006;23(4):607-618.

[38] Ian Max Møller, Abir U. Igamberdiev, Natalia V. Bykova et al. Matrix Redox. Physiology Governs the Regulation of Plant Mitochondrial Metabolism through Posttranslational Protein Modifications, The Plant Cell, 2020,32: 573-594.

[39] Anyonya R. Guntur, Phuong T. Le, Charles R. Farber et al. Bioenergetics During Calvarial Osteoblast Differentiation Reflect Strain Differences in Bone Mass. Endocrinology. 2014; 155(5): 1589-1595.

[40] Peter S. Harris, Samantha R. Roy, Christina Coughlan et al. Chronic ethanol consumption induces mitochondrial protein acetyla- tion and oxidative stress in the kidney. Redox Biology 2015, 6; 33-40.

[41] Pankaj K. Bagul, Parmeshwar B. Katare, Paramesha Bugga et al. SIRT-3 Modulation by Resveratrol Improves Mitochondrial Oxidative Phosphorylation in Diabetic Heart through Deacetylation of TFAM. Cells 2018, 7, 235.

[42] Ian Max Møller, Abir U. Igamberdiev, Natalia V. Bykova et al. Matrix Redox Physiology Governs the Regulation of Plant Mitochondrial Metabolism through Posttranslational Protein Modifications. The Plant Cell, 2020,32: 573-594.

[43] Li TY, Sleiman MB, Li H, et al. The transcriptional coactivator CBP/p300 is an evolutionarily conserved node that promotes longevity in response to mitochondrial stress. Nat Aging. 2021;1(2):165178. doi:10.1038/s43587-020-00025-z.

[44] Aznaurova YB, Zhumataev MB, Roberts TK, Aliper AM, Zhavoronkov AA. Molecular aspects of development and regulation of endometriosis. Reprod Biol Endocrinol. 2014;12:50. Published 2014 Jun 
13. doi:10.1186/1477-7827-12-50.

[45] Yao W, Wang T, Huang F. p300/CBP as a Key Nutritional Sensor for Hepatic Energy Homeostasis and Liver Fibrosis. Biomed Res Int. 2018;2018:8168791. Published 2018 May 15. doi:10.1155/2018/8168791.

[46] Fan L, Li J, Yu Z, Dang X, Wang K. The hypoxia-inducible factor pathway, prolyl-hydroxylase domain protein inhibitors, and their roles in bone repair and regeneration. Biomed Res Int. 2014;2014:239356. doi:10.1155/2014/239356.

\section{Figures}


A
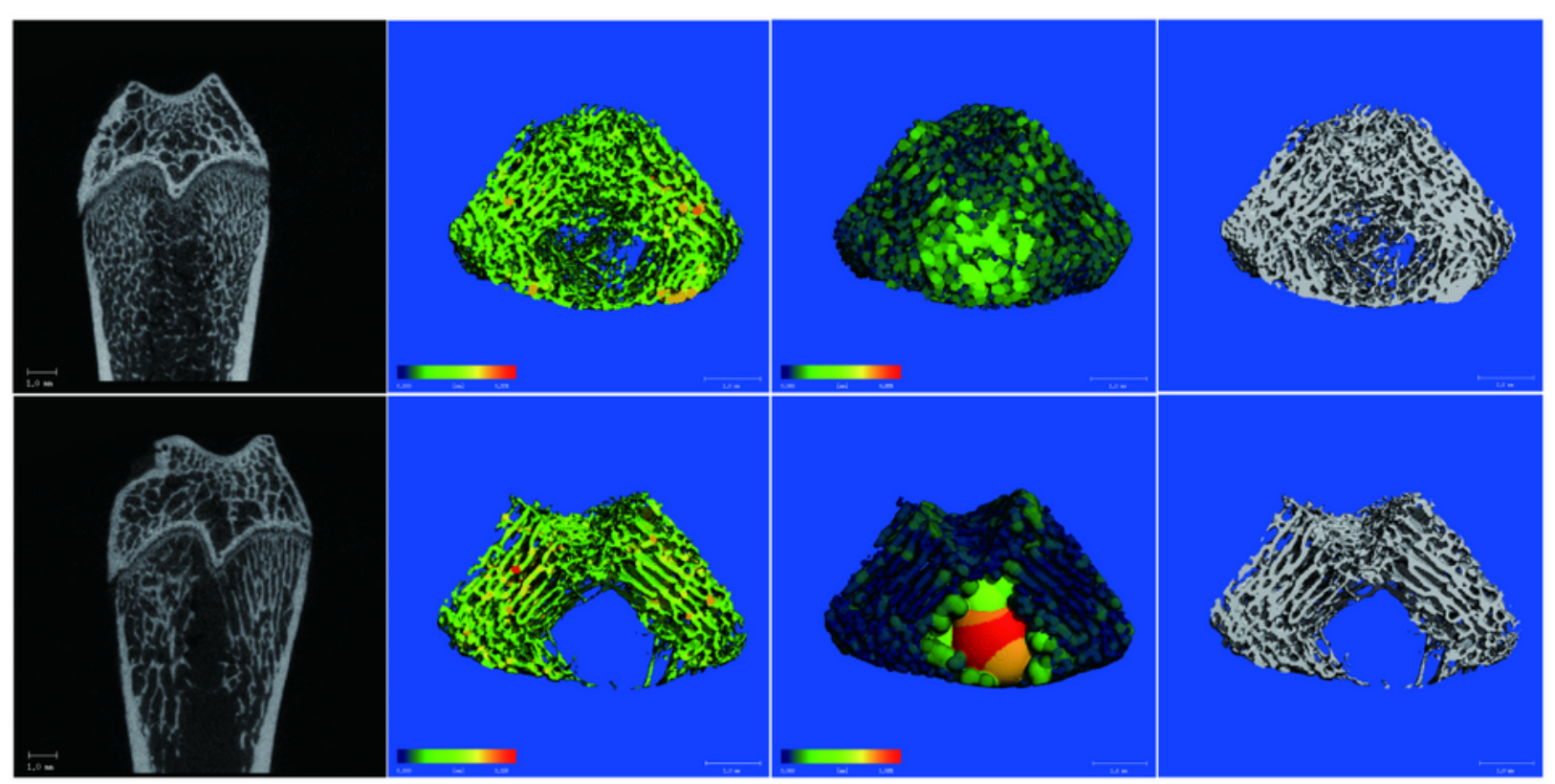

B

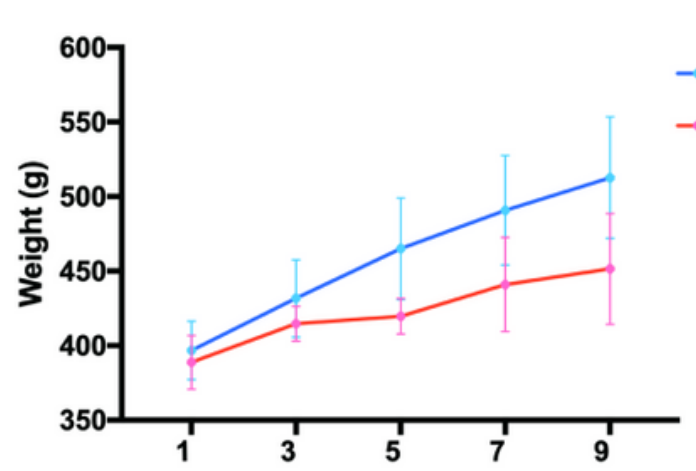

D

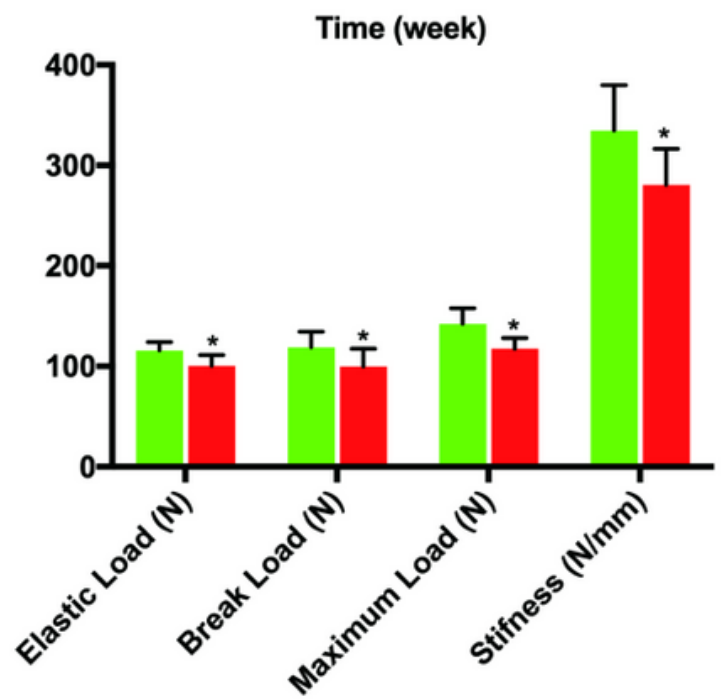

E
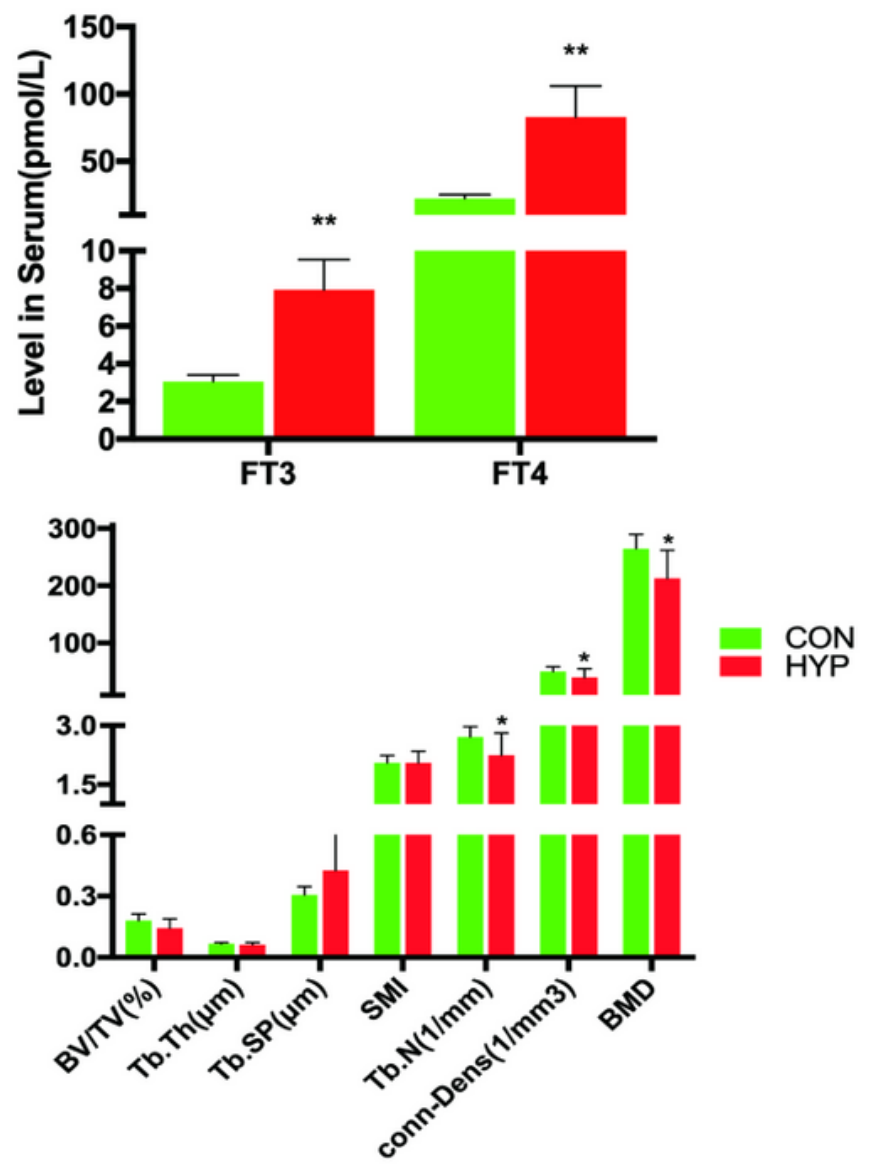

Figure 1

HYP caused bone loss in rats. (A) CT scan view of HYP and CON rats. (B) HYP had passive effect on weight of rats. (C) The level of T3 and T4 in serum in HYP rats. (D) Bone Biochemistry in rats. (E) Parameters of CT scan in rats. $(*: p<0.05 ; * *: p<0.01)$. 
HYP-induced osteoporosis rats

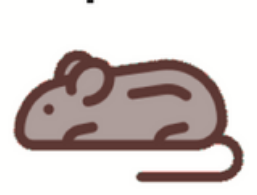

Tissue fractionation
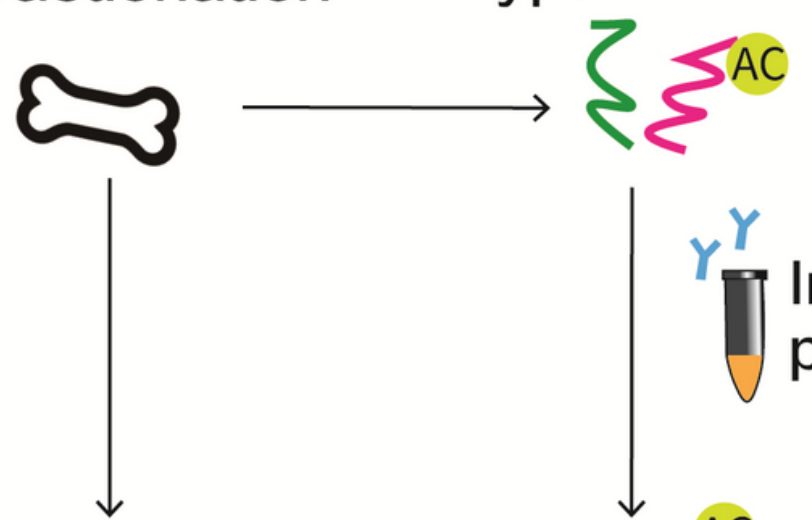

Metabolites identification

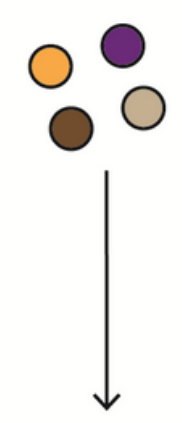

LC-MS analysis
Trypsinization
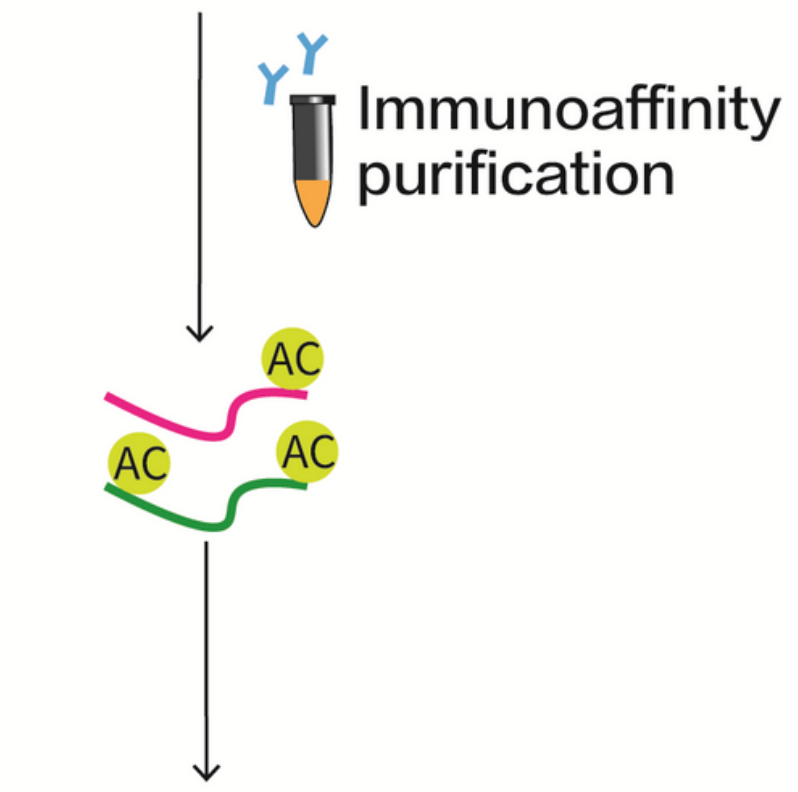

LC-MS analysis

Figure 2

Strategy for Proteomics Analysis of Lysine-Acetylated Proteins and Metabolomics targeted on metabolite of Glycolysis- TCA- Oxidative phosphorylation. 


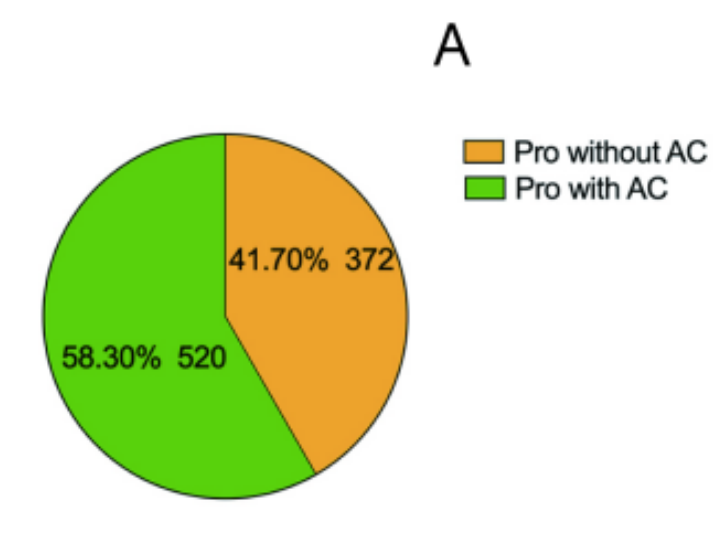

Total $=892$

B

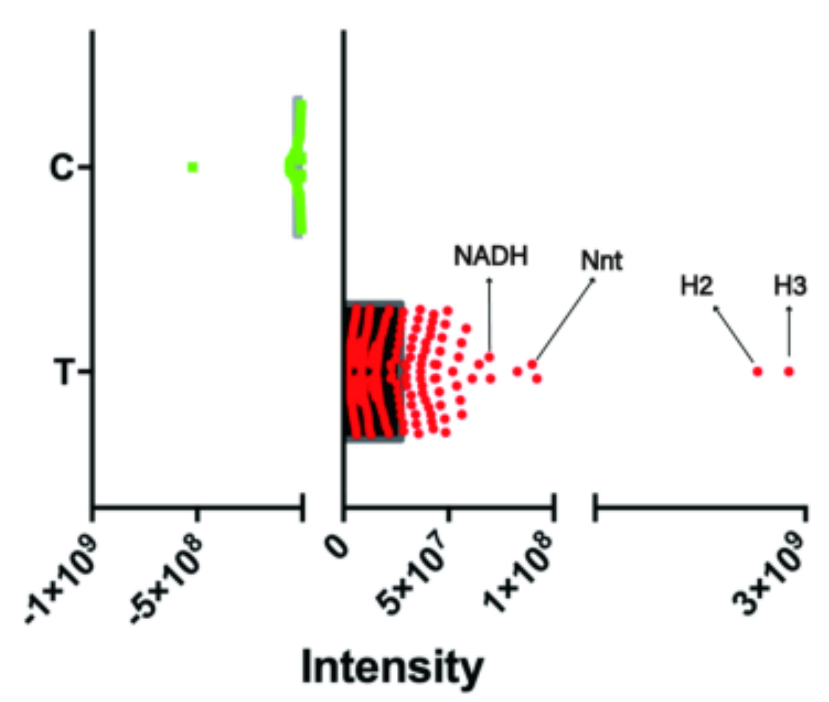

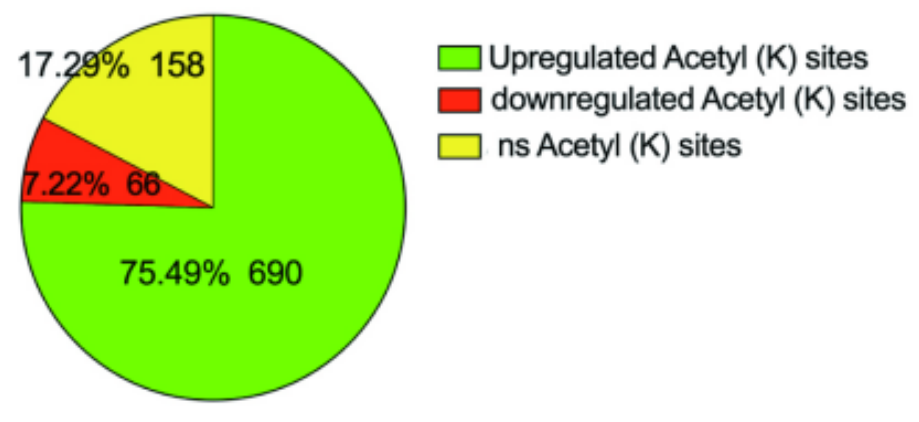

Total $=914$

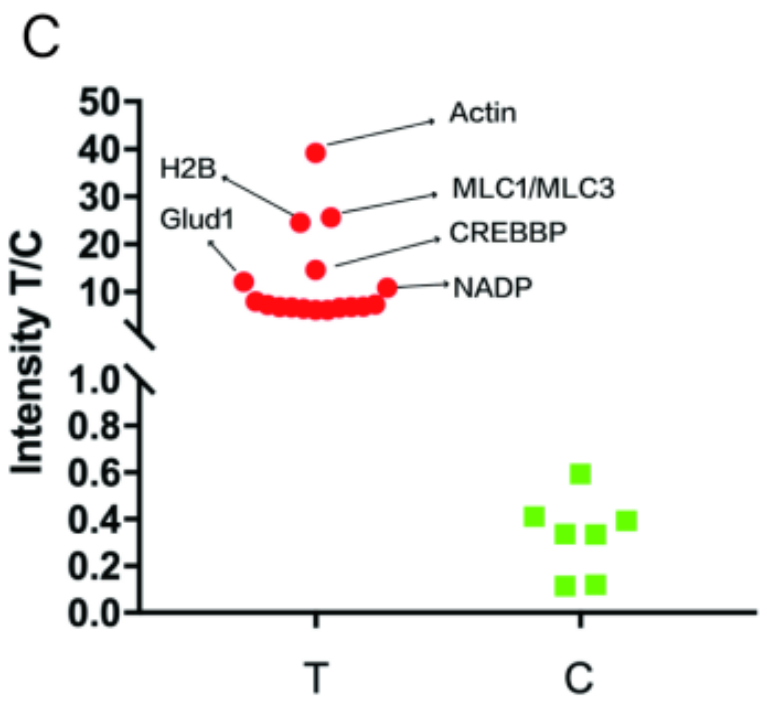

\section{Figure 3}

HYP increase the level of acetylation of different proteins in bone tissue of rats. (A) Differences in acetylation levels in all detected-proteins (ns: not significant); (B) HYP acetylates numbers of proteins; (C) HYP increased the level of acetylation of specific proteins. (AC: Acetylation) 
A

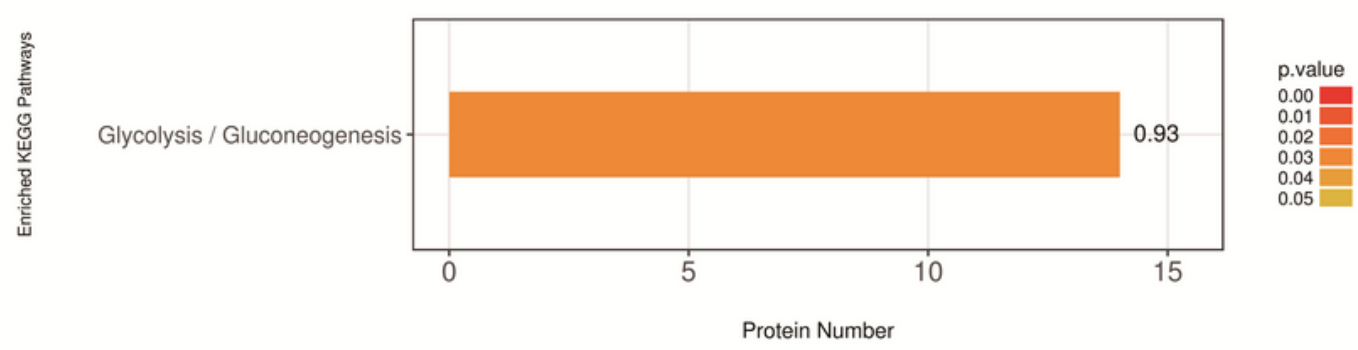

B

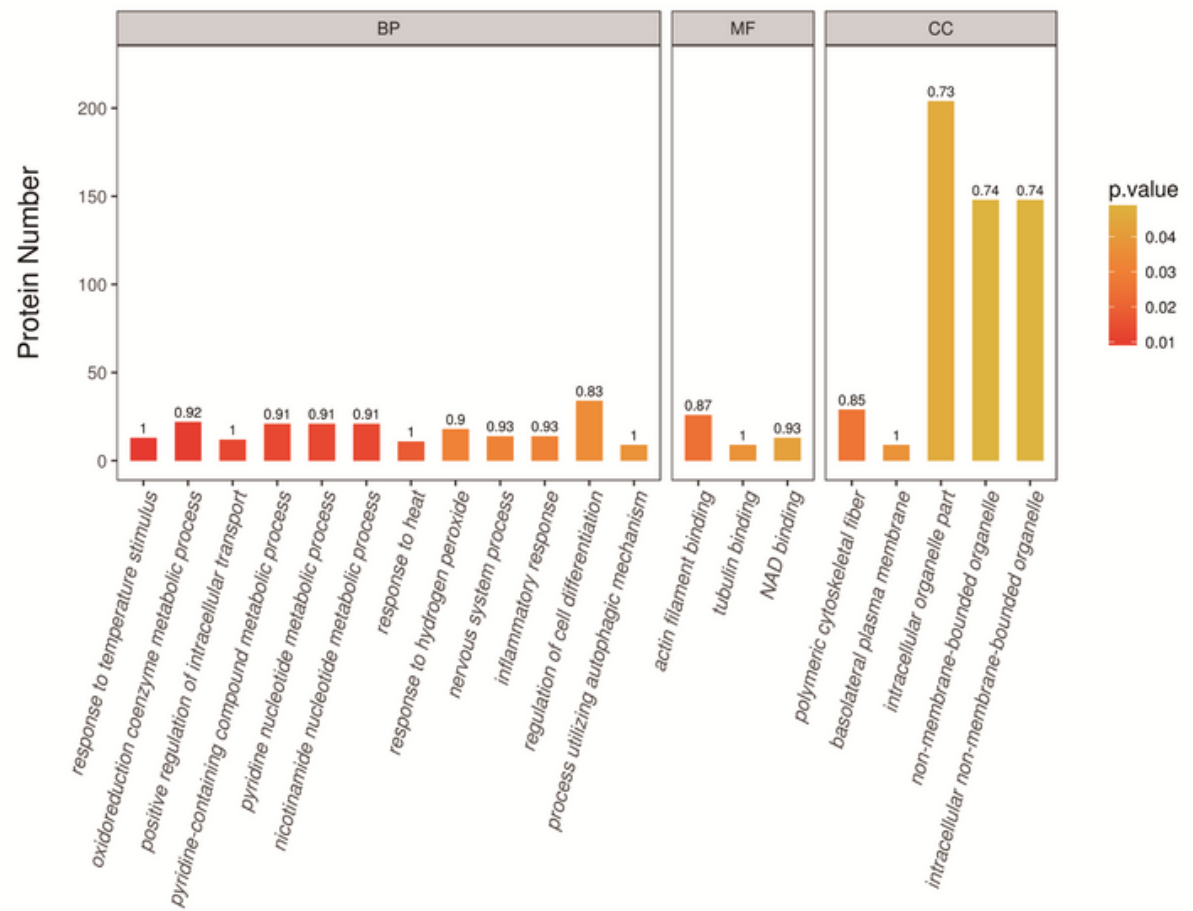

C

\section{Oxidative phosphorylation}

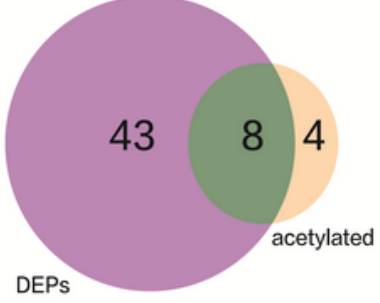

TCA

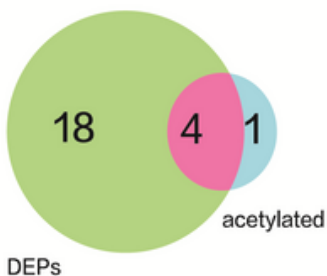

Glycolysis

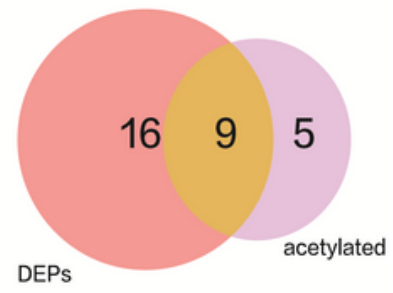

\section{Figure 4}

Acetylome in bone tissue of rats. (A) Enrichment analysis of KEGG pathway of different-acetylated proteins in HYP rats; (B) Enrichment analysis of GO of different-acetylated proteins in HYP rats; (C) The overlap of protein abundance with different levels of acetylation and proteins with different expressions in TCA, Glycolysis and Oxidative phosphorylation (DEPs: different expression proteins). 


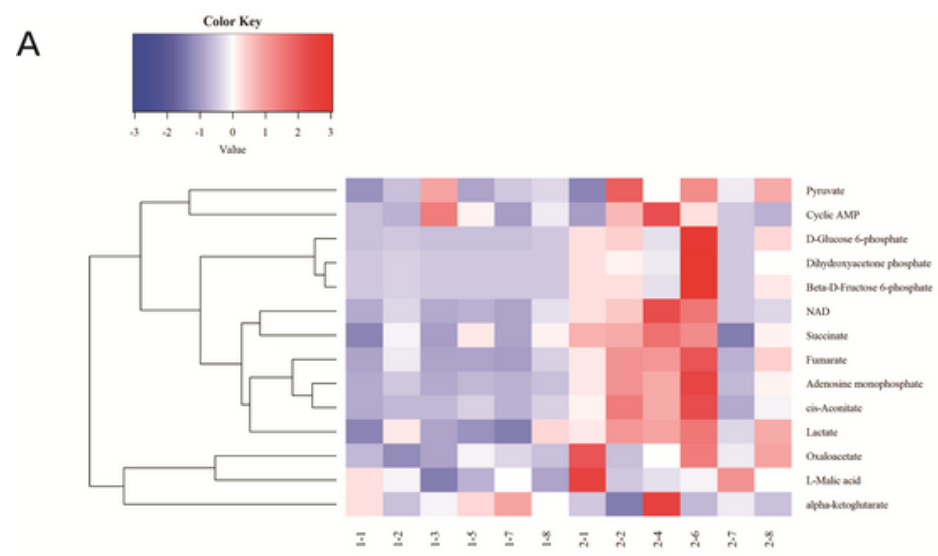

B
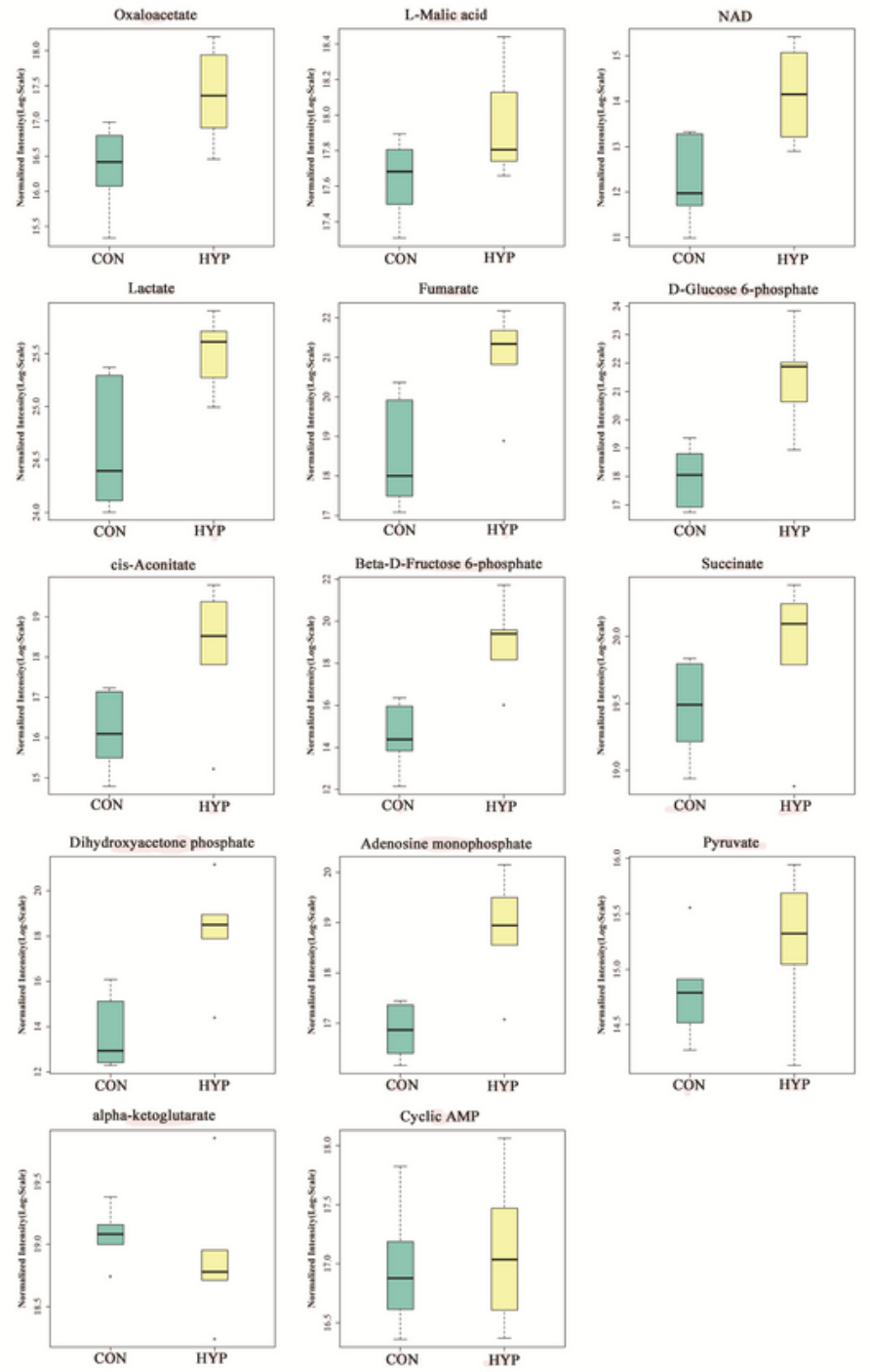

\section{Figure 5}

Diagram of differences in metabolites of rat bone tissue. (A) Hierarchical clustering heat map of metabolites in rats (TREAT: HYP-induced OP group); (B) Boxplot of different-metabolites in rats (Fold change $\leq 0.5$ ). 


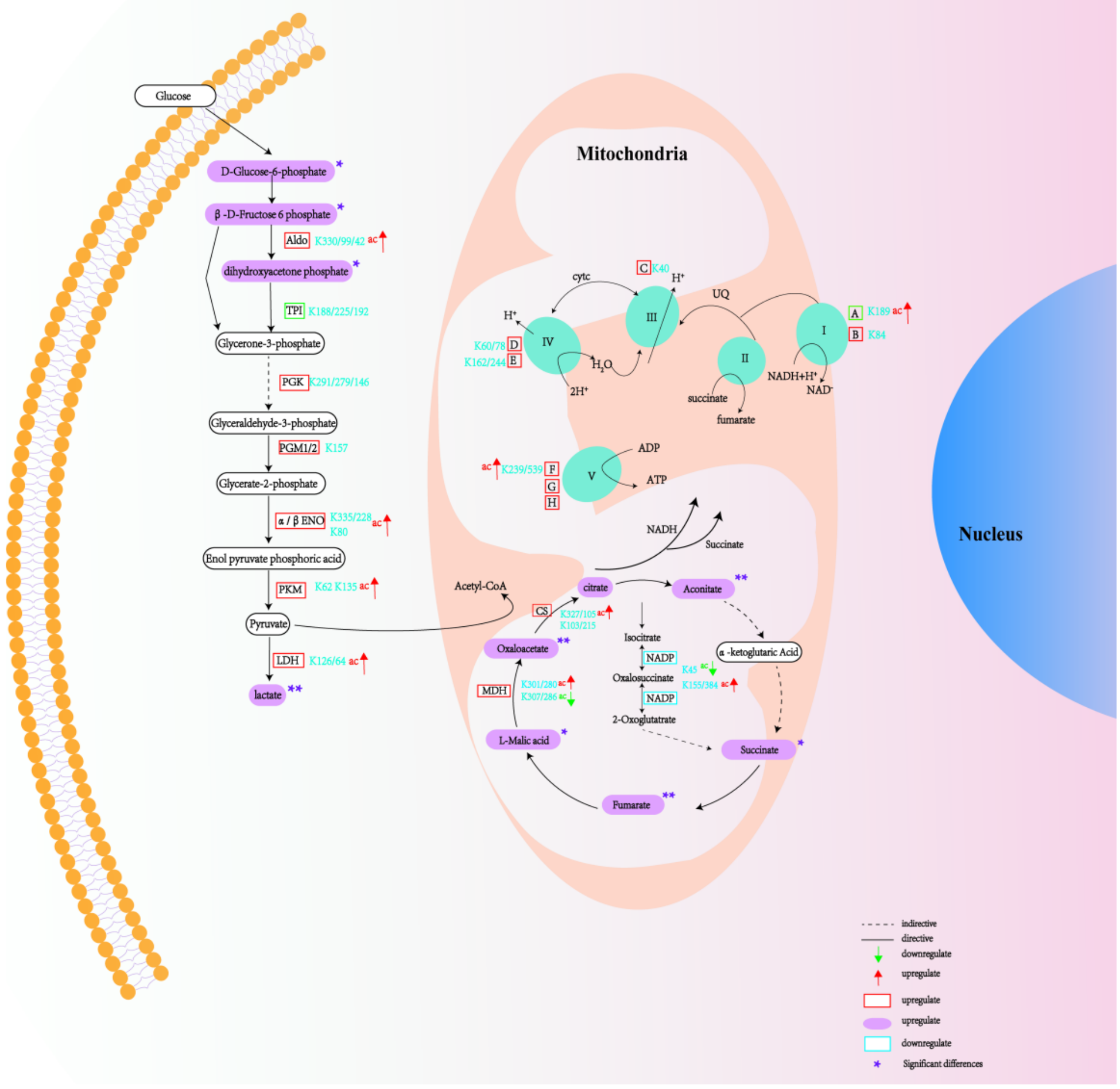

\section{Figure 6}

Glycolysis- TCA- Oxidative phosphorylation in HYP-induced OP rats A: NADH dehydrogenase 1 alpha subcomplex subunit $9 \mathrm{~B}$ : NADH-ubiquinone oxidoreductase $75 \mathrm{kDa}$ subunit C: Cytochrome b-c1 complex subunit $6 \mathrm{D}$ : Cytochrome $\mathrm{c}$ oxidase subunit 4 isoform $1 \mathrm{E}$ : ATP synthase $\mathrm{F}(0)$ complex subunit B1 F: ATP synthase subunit alpha G: ATP synthase subunit $d \mathrm{H}$ : ATP synthase subunit $f$ 


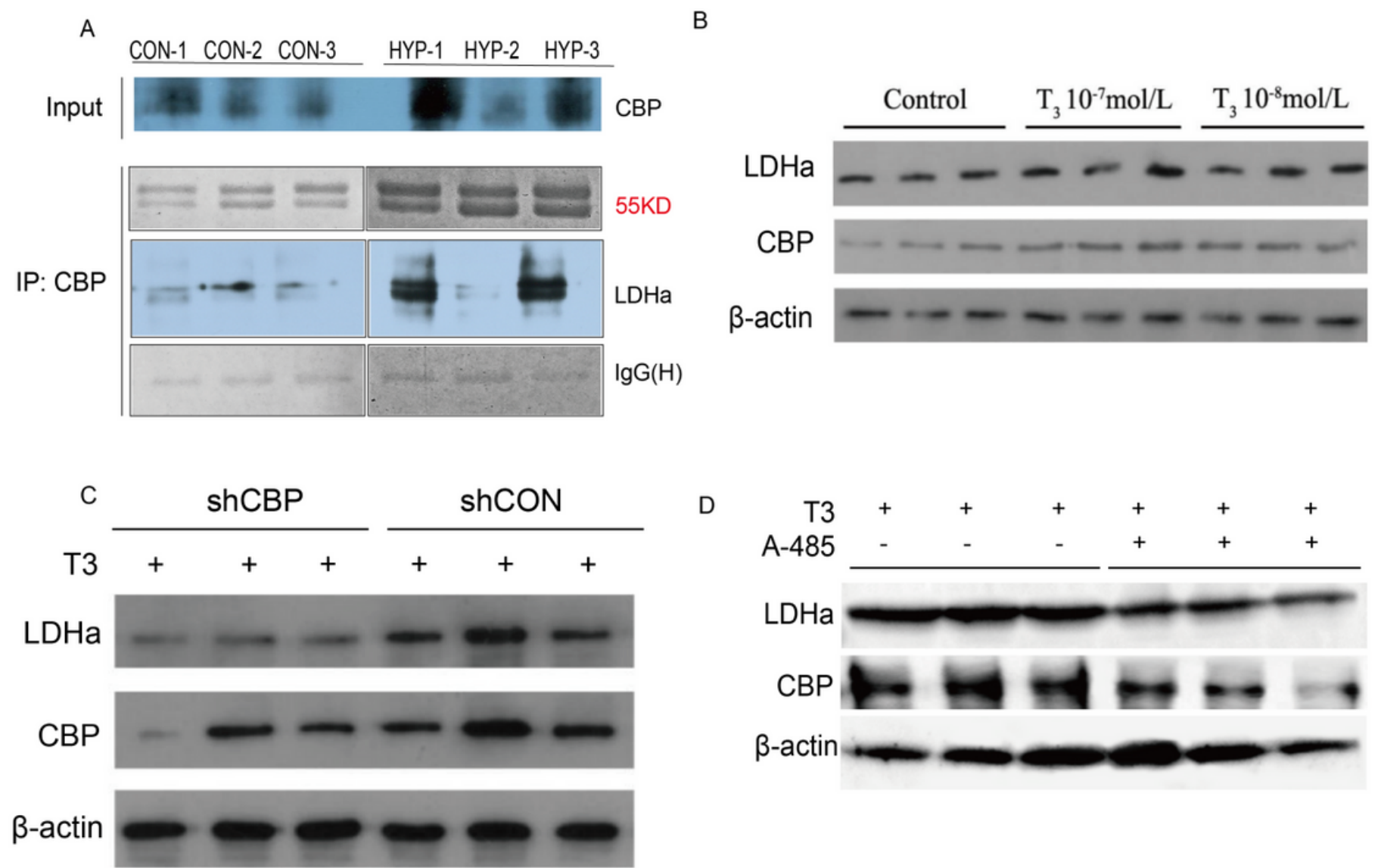

\section{Figure 7}

Expression of CBP and LDHa in HYP rats bone tissues and HYP cell model. (A) Coimmunoprecipitation (ColP) analysis of CBP and LDHa in HYP rats bone tissue. (B) The expression of CBP and LDHa in T3 administration cell model. (C) CBP knockdown reverses LDHa accumulation in T3 administration cell model. (D) A-485 treatment reverses LDHa accumulation in T3 administration model.

\section{Supplementary Files}

This is a list of supplementary files associated with this preprint. Click to download.

- Supplementarytable1.xlsx

- Supplementarytable2.xlsx

- Supplementarytable3.docx

- Supplementarytable4.docx

- FigS1.png

- Figs2.png 